\title{
Genetic description of Manania handi and Manania gwilliami
}

Mark A. Hanson ${ }^{1,2 *}$, Hannah E. Westlake ${ }^{1}$, Louise R. Page ${ }^{1 *}$

1 Department of Biology, University of Victoria, Victoria BC, Canada.

2 Global Health Institute, School of Life Science, École Polytechnique Fédérale de Lausanne (EPFL), Lausanne, Switzerland.

*Corresponding authors: M.A. Hanson (mark.hanson@epfl.ch), L.R. Page (lpage@uvic.ca)

ORCID ID - Hanson: https://orcid.org/0000-0002-6125-3672

\section{Abstract:}

Staurozoa is an intriguing lineage of cnidarians bearing both polypoid and medusoid characters in the adult body plan. Miranda et al. (2016) provided a massive descriptive effort of specimen collection, sequencing, and character evolution. We also recently described the neuromusculature of two staurozoan species: Manania handi and Haliclystus "sanjuanensis." We found that our M. handi samples genetically matched Manania gwilliami samples used in Miranda et al. (2016). Taking advantage of newly-deposited M. gwilliami sequence data, we confirm the identity of our $M$. handi samples, and provide additional sequence data for $M$. handi and $H$. sanjuanensis for future staurozoan identification efforts. 


\section{Introduction:}

Staurozoa (aka "stalked jellyfish") is an ancient lineage of cnidarians with a medusa-like bell attached to a substrate by a polyp-like stalk. This body plan presents an intriguing intermediate between the ancestral anemonelike polyp and the derived medusae of other jellyfish. Whether this body plan is akin to an ancestral pre-medusa ancestor is unclear, as initial analyses of mitochondrial genome data conflicted with nuclear data, placing Staurozoa as either sister to Medusazoa $(1,2)$, sister to Cubozoa alone (3), or as a member of a grand Cubozoa, Scyphozoa, Staurozoa clade (4). Phylogenetic inference using combined mitochondrial and nuclear data also yields different results depending on phylogenetic analysis method (5). Given conflicting phylogenetic signals, the placement of Staurozoa within Cnidaria awaits additional sequence data for more robust comparisons.

As a distinct lineage of cnidarians bearing both polypoid and medusoid characters in the adult body plan, Staurozoa offers a unique opportunity to understand the evolution of other cnidarian groups. Miranda et al. (5) produced a monumental effort of classification, sequencing multiple genetic barcoding regions of diverse staurozoan species. Their phylogenetic inference provided long-needed clarity regarding hypothesized synapomorphic traits used for staurozoan taxonomic description.
We recently provided extensive characterization of the neuromusculature for two Staurozoan species: Manania handi and Haliclystus "sanjuanensis" (6). Notably, Miranda et al. (5) did not include a $M$. handi sample in their analyses, using only two Manania species: M. uchidai and $M$. gwilliami. Manania handi and M. gwilliami are both native to the west coast of North America (7), and genetic type specimens for M. gwilliami used for sequence comparisons in Miranda et al. (5) came from samples collected in California (hereafter $M$. gwilliami (CA)). Curious to support our identification of $M$. handi and H. sanjuanensis, we performed Sanger sequencing of mitochondrial $16 \mathrm{~s}$ and COI loci, and also nuclear 18s (SSU) and ITS (internal transcribed spacer) regions for our specimens.

\section{Results and Discussion:}

Sequence data supported our identification of $H$. sanjuanensis as our specimen consistently clustered with other $H$. sanjuanensis samples in phylogenetic analyses for 16s, COI, 18s, and ITS sequences (Figure 1 and not shown). However, surprisingly we found that all four loci of our $M$. handi specimen were $\geq 99.7 \%$ identical to $M$. gwilliami (CA). Since Miranda et al. (5) and our study (6), additional COI data from M. gwilliami samples collected in 2018 in Bamfield BC Canada were deposited in GenBank; Bamfield is geographically near our sampling location in Victoria BC Canada. We used this opportunity to confirm these putative identifications of $M$. handi and M. gwilliami. 
We found that the three M. gwilliami samples from Bamfield clustered together as a separate group, distinct from our $M$. handi and the included M. gwilliami (CA) sample (Figure 1). While our specimen from Victoria BC is $99.7 \%$ identical at COI when compared to M. gwilliami (CA), our M. handi specimen was only $86.5 \%$ identical to $M$. gwilliami collected in Bamfield. We additionally confirmed our specimen as $M$. handi using characters described in Larson and Fautin (7) (Figure 2), and with Miranda et al. (personal communication). As such, we suggest that the type specimens for $M$. gwilliami used in Miranda et al. (5) were in fact $M$. handi. However, our analysis indeed suggests that M. gwilliami is a distinct species from M. handi, as Manania specimens collected in Victoria and Bamfield differ markedly at their COI locus. To further confirm M. gwilliami as a distinct genetic lineage, further sequence comparisons involving other Manania species should be performed.

Species-level identifications are difficult for lesser-studied marine invertebrates, particularly uncultivable species like staurozoans. By performing an in-depth description in Westlake and Page (6) and generating supporting sequence data (this study), we provide morphological and genetic support for $M$. handi and $M$. gwilliami identification efforts.

\section{Materials and Methods:}

Samples were collected from the intertidal zone adjacent to Chinese Cemetery in Victoria BC, described fully in Westlake and Page (6). DNA was extracted from tissue samples using PrepMan Ultra. We used the 16s, 18s (SSU), COI and ITS primers from Miranda et al. (5). Sanger sequencing was performed by Macrogen USA. Sequences

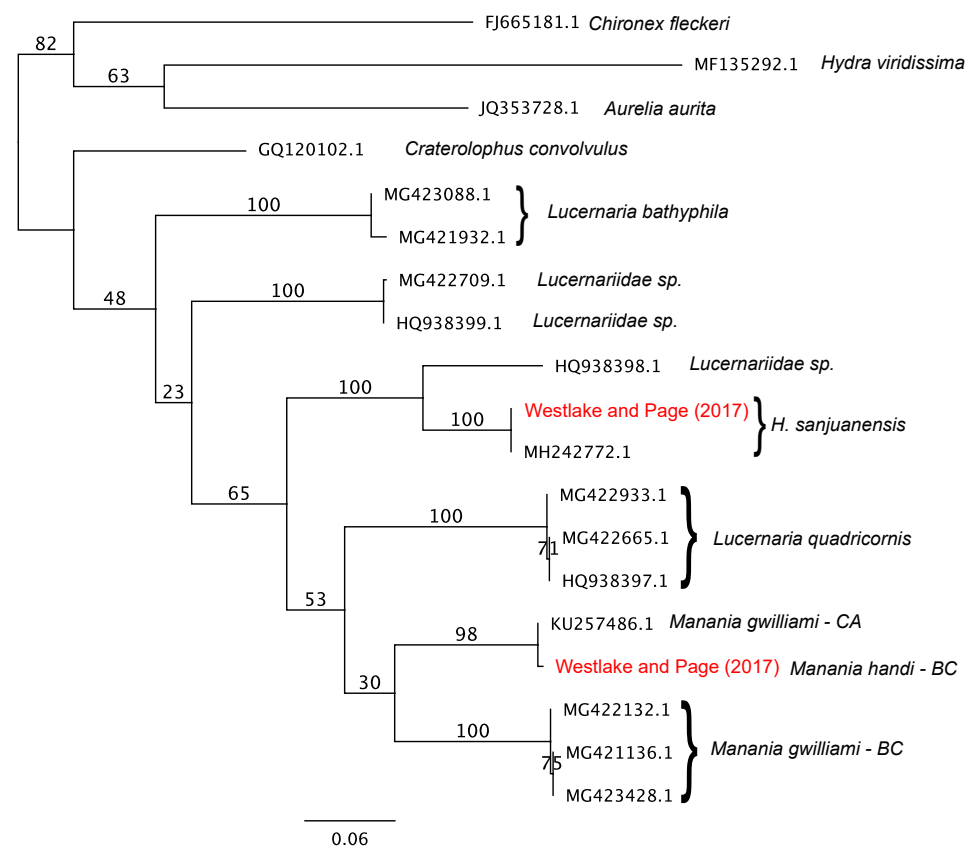

Figure 1: ML phylogeny (100 boostraps) of Staurozoan COI sequence, including samples from Westlake and Page (2017) in red. Notably, M. handi from Victoria (BC) and M. gwilliami (CA) form a distinct clade from M. gwilliami collected in Bamfield (BC). More robust phylogenetic inferences are discussed at length in Miranda et al. (5). GenBank accession numbers are given for pre-existing sequences.
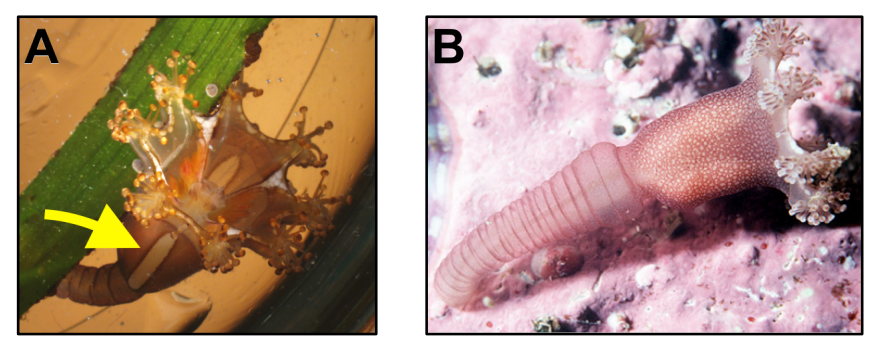

Figure 2: Representative images of $M$. handi (A) and $M$. gwilliami (B). Adult $M$. handi can be distinguished by distinctive "windows" of light-coloured pigment bounded by straight lines along the calyx (arrow), while adult M. gwilliami lacks these windows. The stalk of M. gwilliami is as long or longer than the calyx (7). Manania gwilliami photo by: Thomas Carefoot.

were aligned using MAFFT (8), and phylogenetic analyses were performed in Geneious R10 (9) using the PhyML plugin (10). Sequences generated in this study have been deposited in GenBank under the following accession numbers: MN329738MN329745. 


\section{Acknowledgements:}

HEW and LRP collected, photographed, and identified specimens. MAH sequenced specimens, analyzed data, and wrote this report. All authors read and approved the final report. Tom Carefoot generously provided the photograph of Manania gwilliami used in Figure 2. We owe special thanks to the team at the Centre for Biodiversity Genomics (University of Guelph, Canada) for making their M. gwilliami data publicly available.

\section{References:}

1. Collins A, et al. (2006) Medusozoan phylogeny and character evolution clarified by new large and small subunit rDNA data and an assessment of the utility of phylogenetic mixture models. Syst Biol. doi:10.1080/10635150500433615.

2. Van Iten H, Marques AC, Leme J de M, Pacheco MLAF, Simões MG (2014) Origin and early diversification of the phylum Cnidaria Verrill: Major developments in the analysis of the taxon's proterozoic-cambrian history. Palaeontology. doi:10.1111/pala.12116.

3. Kayal E, Roure B, Philippe H, Collins AG, Lavrov D V. (2013) Cnidarian phylogenetic relationships as revealed by mitogenomics. BMC Evol Biol. doi:10.1186/1471-214813-5.

4. Zapata F, et al. (2015) Phylogenomic analyses support traditional relationships within Cnidaria. PLoS One. doi:10.1371/journal.pone.0139068.

5. Miranda LS, et al. (2016) Systematics of stalked jellyfishes (Cnidaria: Staurozoa). PeerJ. doi:10.7717/peerj.1951.

6. Westlake HE, Page LR (2017) Muscle and nerve net organization in stalked jellyfish (Medusozoa: Staurozoa). J Morphol 278(1):29-49.

7. Larson RJ, Fautin DG (1989) Stauromedusae of the genus Manania (= Thaumatoscyphus ) (Cnidaria, Scyphozoa) in the northeast Pacific, including descriptions of new species Manania gwilliami and Manania handi . Can J Zool. doi:10.1139/z89-219.

8. Katoh K, Standley DM (2013) MAFFT multiple sequence alignment software version 7: Improvements in performance and usability. Mol Biol Evol 30(4):772-780.

9. Kearse M, et al. (2012) Geneious Basic: An integrated and extendable desktop software platform for the organization and analysis of sequence data. Bioinformatics. doi:10.1093/bioinformatics/bts199.

10. Guindon S, et al. (2010) New algorithms and methods to estimate maximumlikelihood phylogenies: Assessing the performance of PhyML 3.0. Syst Biol 59(3):307321. 